\title{
Classificação do Risco de Quedas em Idosos com Least Squares Support Vector Regression Utilizando Sinais Eletromiográficos e Dinamométricos.
}

\author{
Daniele Alves da Silva \\ Centro de Tecnologia \\ Universidade Federal do Piauí \\ Teresina, Piauí \\ d.alves@ufpi.edu.br
}

\author{
Nayra Ferreira Lima Castelo Branco \\ Centro de Ensino Unificado do Piauí \\ Teresina, Piauí \\ nayra.ferreira@ceupi.edu.br
}

\author{
Laiana Sepúlveda de Andrade Mesquita \\ Centro de Ciências da Saúde \\ Universidade Estadual do Piauí \\ Teresina, Piauí \\ laianasepulveda@ccs.uespi.br
}

\author{
Hermes Manoel Galvão Castelo Branco \\ Centro de Tecnologia \\ Universidade Federal do Piauí \\ Teresina, Piauí \\ hermescb@ufpi.edu.br
}

\author{
Luan Marinho Morais Pereira \\ Centro de Tecnologia e Urbanismo \\ Universidade Estadual do Piauí \\ Teresina, Piauí \\ luanpereira@aluno.uespi.br
}

\begin{abstract}
Resumo - A determinação do risco de cair é de suma importância na assistência à saúde do idoso, pois a ocorrência de quedas nessa população trazem consequências em vários aspectos. Ferramentas de aprendizado de máquinas têm sido cada vez mais empregadas com este fim. Portanto, o objetivo deste estudo foi investigar a viabilidade da utilização de sinais eletromiográficos e dinamométricos na classificação do risco de quedas em idosos via modelo least squares support vector regression (LSSVR). Trinta e um voluntários idosos foram avaliados com a Escala de Equilíbrio de Berg (EEB), eletromiografia e dinamometria do membro inferior dominante. Para o modelo LSSVR foram utilizados kernels do tipo linear, polinomial e radial basis function (RBF), além de validações cruzadas pelos métodos leave one out e $K$-fold. Ambos os sinais apresentaram erros médios baixos na maioria das execuções realizadas. Dessa forma, verificou-se que é possível classificar o risco de quedas em idosos por meio de sinais eletromiográficos e dinamométricos aplicados ao modelo LSSVR.
\end{abstract}

Palavras-Chaves-Escala de Equilibrio de Berg, Idosos, Risco de Quedas, Inteligência Computacional, Least Squares Support Vector Regression.

\section{INTRODUÇÃO}

O expressivo aumento do contingente populacional de idosos nos últimos anos tem tido grande relevância no contexto da saúde pública [1], uma vez que a ocorrência de quedas, comum nessa faixa etária, traz consequências em vários aspectos. Em 2011, um grupo de pesquisadores averiguou 100 municípios em 23 estados brasileiros e constatou que $27,6 \%$ dos idosos caíram no período de um ano [2]. A hospitalização desses idosos caidores é corriqueira, dada a fragilidade do organismo nesta fase da vida. Estima-se que as despesas médicas atribuídas a quedas foram de aproximadamente 50 bilhões de dólares nos Estados Unidos, no ano de 2015 [3].

Em paralelo à questão econômica, há de se considerar o impacto nas relações sociais, na saúde e na qualidade de vida desses idosos. A pesquisa de Siqueira et al, 2011 [2], também identificou que uma parcela de $11 \%$ dos idosos que caíram sofreram fraturas. A mobilidade reduzida, seja por processos cirúrgicos, seja pelo medo de cair novamente, causa adaptações no sistema musculoesquelético que facilitam que uma nova queda aconteça, além de afetar a execução de atividades da vida diária [4].

Diante das consequências que os episódios de quedas acarretam, fica evidente que a prevenção é um possível caminho para evitá-las; portanto, determinar o risco de cair, a fim de identificar os fatores envolvidos, é de suma importância na assistência ao idoso [5]. Isto posto, os profissionais da saúde dispõem de muitas ferramentas elaboradas com este propósito, como por exemplo, o Timed Up and Go Teste (TUGT), a Escala de Equilíbrio de Berg (EEB) e a escala de Tinetti. Estes, porém, são métodos subjetivos [6] e que apresentam limitações quanto à eficiência de predição para grupos específicos de idosos [5,7].

Nesse contexto, é oportuno que se procure melhorar ou até mesmo desenvolver novas e mais acuradas metodologias de predição com o emprego da tecnologia. Ferramentas de aprendizado de máquinas tiveram grande participação nos estudos com esse escopo, publicados na última década [8-10]. Apesar disso, não foram encontradas pesquisas que empregassem o modelo least squares support vector regression (LSSVR) como instrumento de predição de risco de quedas em idosos. Esta ferramenta é um método de kernel para regressão não linear cujos parâmetros são estimados pelo estimador de mínimos quadrados e, portanto, mantém algumas de suas características como a facilidade de implementação por meio de uma expressão analítica e otimalidade do estimador [11].

De forma semelhante, vários estudos empregaram sensores inerciais para obter sinais para classificação do 
risco de quedas [12,13], no entanto poucos empregaram a eletromiografia como dispositivo de aquisição, apesar da clara associação entre a co-contração muscular e o risco de cair [14].

Portanto, o objetivo deste estudo é investigar a viabilidade da utilização de sinais eletromiográficos e dinamométricos na predição do risco de quedas em idosos por meio de um algoritmo LSSVR. Outro aspecto investigado no estudo é averiguar se biosinais, no caso sinais eletromiográficos, são tão relevantes quanto os sinais de força para a predição do risco de quedas em idosos. A principal contribuição deste trabalho é o desenvolvimento de uma ferramenta de estimação de riscos de quedas que diminua a subjetividade das avaliações. Por meio da análise de biosinais. Outra contribuição relevante é a comparação entre as estimativas realizadas a partir de sinais de força e aquelas realizadas dispondo de sinais de eletromiografia, uma vez que este tipo de comparação ainda não foi encontrada na literatura correlata. Como contribuição adicional, além da aplicação de LSSVR, investigamos a aplicação de sinais obtidos em músculos diferentes daqueles empregados em [14], bem como a extração de atributos dos sinais ainda não utilizadas em outros trabalhos para classificar o risco de quedas. Destaca-se que é levado a cabo neste artigo um estudo amplo sobre os atributos mais relevantes dos sinais aquisitados para a avaliação do risco de queda.

O restante deste trabalho está organizado nas seguintes seções: II metodologia; III Resultados; IV Discussões; e V Conclusões.

\section{METODOLOGIA}

\section{A. Delineamento do Estudo}

Este estudo foi desenvolvido entre os meses de novembro de 2020 e fevereiro de 2021, após aprovação pelo Comitê de Ética em Pesquisa (CEP) da Universidade Estadual do Piauí (UESPI) e posterior autorização de uma clínica privada para utilização do ginásio como local para coleta dos dados.

Foram incluídas pessoas com idade igual ou superior a 60 anos e que concordaram em participar da pesquisa, espontaneamente, por meio da assinatura do Termo de Consentimento Livre e Esclarecido (TCLE), conforme a resolução n466/2012 do Conselho Nacional de Saúde. Foram excluídos os participantes com idade abaixo de 60; portadores de vestibulopatias ou comprometimentos de natureza ortopédica, cardiovascular, psiquiátrica ou neurológica que inviabilizassem a execução de todas as etapas da avaliação; bem como aqueles que não concordaram em participar do estudo.

A amostra, por conveniência e dificuldade de coleta de dados em período pandêmico, foi composta por 30 idosos voluntários (8 homens e 22 mulheres), recrutados da comunidade. Seguindo todos os protocolos sanitários instituídos por conta da pandemia de COVID-19, os participantes passaram por uma entrevista inicial, na qual foram coletados dados demográficos e em seguida tiveram o equilíbrio avaliado por meio da Escala de Equilíbrio de Berg.

A EEB é uma ferramenta bastante utilizada na prática clínica para avaliar o equilíbrio funcional de idosos. A escala é composta de 14 itens baseados em atividades da vida diária, onde o avaliador atribui uma pontuação que varia de 0 a 4 pontos de acordo com o desempenho do indivíduo na execução de cada item. $\mathrm{O}$ escore final varia de 0 a 56 pontos e é inversamente proporcional ao risco de cair, ou seja, quanto maior a pontuação menor o risco de o avaliado vir a sofrer quedas e vice-versa. [15]

\section{B. Avaliação de Força e Eletromiografia}

Posteriormente, foi realizada a avaliação por meio da eletromiografia (EMG). Para a aquisição de sinal elétrico foi utilizado o sistema de aquisição de sinais EMG System SAS1000 V8 (EMG System, Brasil) com capacidade para coleta de até seis canais simultâneos, frequência de amostragem de $2000 \mathrm{~Hz}$ e filtro passa banda de 20-500 $\mathrm{Hz}$. O equipamento conta ainda com conversor analógicodigital de 12 bits trabalhando com uma frequência de amostragem de $2 \mathrm{kHz}$ em cada canal, evitando o problema de aliasing. Para a avaliação da força empregou-se uma célula de carga (dinamômetro), do mesmo fabricante, do tipo Tração/Compressão com capacidade de leitura de até $200 \mathrm{Kgf}$ de forma sincronizada com os registros do eletromiógrafo.

Após a assepsia da pele, eletrodos bipolares autoadesivos foram posicionados em pontos motores de quatro músculos do membro inferior dominante de cada participante: vasto lateral, bíceps femoral, tibial anterior e gastrocnêmio. Esses músculos foram escolhidos por terem grande influência na manutenção do equilíbrio postural estático. Um eletrodo de referência foi fixado no epicôndilo lateral do cotovelo do membro superior dominante.

O equipamento utilizado neste estudo permite a captação de sinal pelos eletrodos e pela célula de carga de forma simultânea. Assim, a aquisição dos sinais tanto elétricos quanto dinamométricos, a serem posteriormente fornecidos como entradas para o modelo LSSVR, se deu por meio da solicitação de uma contração isométrica de cada músculo mencionado, como descrito a seguir.

Para o músculo vasto lateral, foi solicitado que o participante sentasse em uma cadeira e apoiasse bem as costas, para evitar compensações de outros músculos, com o joelho em um ângulo de $90^{\circ}$. Uma extremidade da célula de carga foi posicionada no tornozelo do voluntário com o auxílio de uma correia e a outra foi presa a uma estrutura metálica estável atrás do participante. Ao idoso foi solicitado o movimento de esticar o joelho e a manutenção desta posição por 10 segundos.

Para o músculo bíceps femoral, manteve-se a posição sentada do participante e fixou-se o dinamômetro em uma estrutura metálica estável à frente do participante. Solicitou-se então o movimento de dobrar o joelho, mantendo-se a contração por 10 segundos.

Para o músculo tibial anterior, o participante foi posicionado em decúbito dorsal em um tablado com uma extremidade do dinamômetro posicionada no pé com o auxilio da correia e a outra extremidade fixada em estrutura metálica estável anterior à planta do pé do voluntário. Foi solicitado que o idoso realizasse o movimento de levar a ponta do pé para cima e mantivesse a contração por 10 segundos.

Para o músculo gastrocnêmio, manteve-se a posição em decúbito dorsal e o dinamômetro teve uma 
extremidade fixada em estrutura metálica estável posterior ao topo da cabeça do voluntário. Foi solicitado que o voluntário realizasse o movimento de levar a ponta do pé para baixo e mantivesse por 10 segundos.

\section{Processamento do Sinal}

Após a coleta, os dados foram analisados, sendo excluídos dados em que ocorreram problemas no processo de aquisição. Foram obtidos sinais de todos os músculos durante a realização de cada uma das atividades. Em cada sinal coletado, foram calculados a seguintes estatísticas descritivas: valor máximo, mínimo, média e desvio padrão. Foram extraídos ainda os seguintes atributos no domínio do tempo:

\section{a) RMS (Root Mean Square)}

$\mathrm{O}$ valor RMS é representado matematicamente como

$$
R M S=\sqrt{\frac{1}{N} \sum_{i=1}^{N} x_{i}^{2}}
$$

em que, $x_{i}$ representa o sinal EMG no segmento $i$ e $N$ representa o tamanho (ou seja, o número de amostras) do sinal. Este valor mantém uma relação com a força da contração muscular sem fadiga [16], refletindo o nível de atividade fisiológica da unidade motora durante a contração [17]. Foi calculado o valor RMS dos sinais coletados em todos os músculos durante a realização de todas as atividades.

\section{b) Energia}

Representada matematicamente como

$$
E=\sum_{i=1}^{N} X_{i} \mathrm{v}^{2},
$$

esta medida apresenta uma relação com o potencial de ação das unidades motoras durante a contração muscular [18]. A energia foi calculada para todos os sinais coletados em todos os músculos durante a realização de todas as atividades

\section{c) AAC (Average Amplitude Change)}

$\mathrm{O}$ atributo $\mathrm{AAC}$, representada matematicamente como

$$
A A C=\frac{1}{N} \sum_{i=1}^{N-1}\left|x_{i+1}-x_{i}\right|,
$$

é uma medida de complexidade do sinal, sendo definida como a média do comprimento cumulativo da forma de onda do EMG [16]. Mais uma vez, calculou-se o AAC de todos os sinais.

\section{d) LOGD (Log Detector)}

O LOGD representa um detector não linear que fornece uma estimativa da força de contração muscular, expresso matematicamente em (4) [16]. Por fim, calculouse o LOGD de todos os sinais coletados em todos os músculos.

$$
\log =\exp \left(\frac{1}{N} \sum_{i=1}^{N} \log \left(\left|x_{i}\right|\right)\right)
$$

Depois de extraídos os atributos, os dados foram normalizados e aplicados ao como entradas ao modelo LSSVR. Foram realizados três experimentos variando o conjunto de atributos e utilizando os kernels linear, polinomial e radial basis function (RBF), a fim de observar o comportamento dos resultados em cada configuração. As execuções empregaram ainda validação cruzada por leave one out e $k$-folds de tamanhos $2,4,5,6$ e 8. Ademais, nessas configurações já mencionadas, foram realizados testes considerando três grupos de atributos obtidos dos sinais de EMG como entradas. Os mesmos três grupos de atributos obtidos dos dados de força foram utilizados como entradas.

Cada teste foi realizado 500 vezes e a cada repetição, 25 amostras eram selecionadas para participarem do processo de treinamento/validação e 6 amostras eram selecionadas para teste. Destaca-se que uma vez selecionado o conjunto de treinamento/validação e teste; todas as variações de conjuntos de atributos de entrada, tipos de sinais de entrada, tipo de kernel utilizados e quantidade de partições eram realizadas considerando o mesmo conjunto, repetindo-se o processo de escolha de amostras para compor grupos de treinamento/validação e teste por 500 vezes.

A EEB foi utilizada como padrão ouro para classificar os idosos com baixo ou alto risco de cair e a pontuação obtida por cada idoso na escala serviu de base para avaliar o desempenho do algoritmo LSSVR. Para isso foi necessário arredondar as saídas obtidas pelo algoritmo, já que estas e as pontuações da EEB são dadas em números inteiros.

\section{RESULTADOS}

Foram avaliados 30 idosos ( 8 do sexo masculino e 22 do sexo feminino) com idade média de 71,50 anos. Dentre os participantes, 6 foram classificados pela Escala de Equilíbrio de Berg com alto risco de quedas e 24 foram rotulados com baixo risco. A Tab. I apresenta os dados demográficos extraídos da entrevista inicial com os participantes do estudo.

\section{A. Conjunto 1: Todos os Atributos}

$\mathrm{Na}$ Tab. II encontram-se os resultados obtidos com a utilização do modelo LSSVR considerando todos os oito atributos extraídos dos sinais de EMG (máximo, mínimo, média, desvio padrão, RMS, energia, LOGD e AAC) para cada um dos 4 músculos durante os 4 exercícios, produzindo um vetor de atributos com $8 \times 4 \times 4=128$ componentes. Observou-se que, os kernels linear e RBF obtiveram melhores desempenhos de teste, apresentando erros mais baixos que o kernel polinomial.

TABELA I. CARACTERÍSTICAS DEMOGRÁFICAS DOS IDOSOS AVALIADOS.

\begin{tabular}{|c|c|}
\hline Variável & Média \pm Desvio Padrão \\
\hline Idade & $71,500 \pm 5,270$ \\
\hline Peso & $70,866 \pm 18,214$ \\
\hline Altura & $159,033 \pm 7,131$ \\
\hline
\end{tabular}


TABELA II. ERROS MÉDIOS OBTIDOS PELO MODELO LSSVR COM SINAL DE EMG PARA CLASSIFICAÇÃO DO RISCO DE QUEDAS UTILIZANDO TODOS OS ATRIBUTOS CALCULADOS.

\begin{tabular}{|c|c|c|c|c|}
\hline \multirow{2}{*}{ Kernel } & \multirow{2}{*}{\multicolumn{2}{|c|}{$\begin{array}{c}\text { Cross- } \\
\text { Validation }\end{array}$}} & \multicolumn{2}{|c|}{ ERRO MÉDIO } \\
\hline & & & TREINO & TESTE \\
\hline \multirow{6}{*}{$\begin{array}{l}\mathbf{L} \\
\mathbf{I} \\
\mathbf{N} \\
\mathbf{E} \\
\mathbf{A} \\
\mathbf{R}\end{array}$} & \multicolumn{2}{|c|}{ Leave One Out } & $0,147 \pm 0,135$ & $0,195 \pm 0,172$ \\
\hline & \multirow{5}{*}{ K-FOLD } & 2 & $0,152 \pm 0,145$ & $0,195 \pm 0,186$ \\
\hline & & 4 & $0,149 \pm 0,141$ & $0,205 \pm 0,175$ \\
\hline & & 5 & $0,148 \pm 0,138$ & $0,199 \pm 0,181$ \\
\hline & & 6 & $0,148 \pm 0,138$ & $0,204 \pm 0,172$ \\
\hline & & 8 & $0,147 \pm 0,138$ & $0,193 \pm 0,163$ \\
\hline \multirow{6}{*}{$\begin{array}{l}\mathbf{P} \\
\mathbf{O} \\
\mathbf{L} \\
\mathbf{I} \\
\mathbf{N} \\
\mathbf{O} .\end{array}$} & \multicolumn{2}{|c|}{ Leave One Out } & \multicolumn{2}{|c|}{$\begin{array}{l}\text { Não forneceu resultado numérico } \\
\text { válido }\end{array}$} \\
\hline & \multirow{5}{*}{ K-FOLD } & 2 & $0,145 \pm 0,131$ & $0,266 \pm 0,338$ \\
\hline & & 4 & $0,145 \pm 0,131$ & $0,237 \pm 0,266$ \\
\hline & & 5 & $0,143 \pm 0,130$ & $0,306 \pm 0,421$ \\
\hline & & 6 & $0,145 \pm 0,131$ & $0,237 \pm 0,253$ \\
\hline & & 8 & $0,144 \pm 0,131$ & $0,211 \pm 0,189$ \\
\hline \multirow{6}{*}{$\begin{array}{l}\mathbf{R} \\
\mathbf{B} \\
\mathbf{F}\end{array}$} & \multicolumn{2}{|c|}{ Leave One Out } & $0,145 \pm 0,132$ & $0,198 \pm 0,171$ \\
\hline & \multirow{5}{*}{ K-FOLD } & 2 & $0,144 \pm 0,131$ & $0,198 \pm 0,171$ \\
\hline & & 4 & $0,144 \pm 0,132$ & $0,192 \pm 0,166$ \\
\hline & & 5 & $0,145 \pm 0,131$ & $0,195 \pm 0,175$ \\
\hline & & 6 & $0,145 \pm 0,131$ & $0,194 \pm 0,170$ \\
\hline & & 8 & $0,144 \pm 0,131$ & $0,192 \pm 0,165$ \\
\hline
\end{tabular}

$\mathrm{Na}$ Tab. III estão reportados os resultados obtidos com a utilização do modelo LSSVR considerando todos os atributos extraídos dos dados da dinamometria (máximo, mínimo, média, desvio padrão, RMS, energia, LOGD e AAC) para cada um dos 4 exercícios, conduzindo a um vetor de atributos com $8 \times 4=32$ entradas. O kernel polinomial apresentou erros maiores que os demais, tanto no treino quanto na etapa de testes. Por outro lado, o kernel RBF foi o que apresentou menores erros no teste e o kernel linear apresentou resultados tanto da etapa de treino quanto de teste bem próximos.

TABELA III. ERROS MÉDIOS OBTIDOS PELO MODELO LSSVR COM SINAL DE FORCA PARA CLASSIFICAC̃̃̃O DO RISCO DE QUEDAS UTILIZANDO TODOS OS ATRIBUTOS CALCULADOS.

\begin{tabular}{|c|c|c|c|c|}
\hline \multirow{2}{*}{ Kernel } & \multirow{2}{*}{\multicolumn{2}{|c|}{$\begin{array}{c}\text { Cross- } \\
\text { Validation }\end{array}$}} & \multicolumn{2}{|c|}{ ERRO MÉDIO } \\
\hline & & & TREINO & TESTE \\
\hline \multirow{6}{*}{$\begin{array}{l}\mathbf{L} \\
\mathbf{I} \\
\mathbf{N} \\
\mathbf{E} \\
\mathbf{A} \\
\mathbf{R}\end{array}$} & \multicolumn{2}{|c|}{ Leave One Out } & $0,207 \pm 0,242$ & $0,240 \pm 0,222$ \\
\hline & \multirow{5}{*}{ K-FOLD } & 2 & $0,207 \pm 0,241$ & $0,238 \pm 0,225$ \\
\hline & & 4 & $0,209 \pm 0,243$ & $0,229 \pm 0,216$ \\
\hline & & 5 & $0,207 \pm 0,242$ & $0,235 \pm 0,217$ \\
\hline & & 6 & $0,210 \pm 0,246$ & $0,227 \pm 0,210$ \\
\hline & & 8 & $0,207 \pm 0,240$ & $0,238 \pm 0,230$ \\
\hline \multirow{6}{*}{$\begin{array}{c}\mathbf{P} \\
\mathbf{O} \\
\mathbf{L} \\
\mathbf{I} \\
\mathbf{N} \\
\mathbf{O} .\end{array}$} & \multicolumn{2}{|c|}{ Leave One Out } & $0,151 \pm 0,144$ & $0,298 \pm 0,298$ \\
\hline & \multirow{5}{*}{ K-FOLD } & 2 & $0,151 \pm 0,142$ & $0,601 \pm 0,933$ \\
\hline & & 4 & $0,152 \pm 0,145$ & $0,296 \pm 0,299$ \\
\hline & & 5 & $0,150 \pm 0,143$ & $0,345 \pm 0,391$ \\
\hline & & 6 & $0,154 \pm 0,147$ & $0,291 \pm 0,296$ \\
\hline & & 8 & $0,153 \pm 0,146$ & $0,279 \pm 0,278$ \\
\hline \multirow{6}{*}{$\begin{array}{l}\mathbf{R} \\
\mathbf{B} \\
\mathbf{F}\end{array}$} & \multicolumn{2}{|c|}{ Leave One Out } & $0,151 \pm 0,143$ & $0,235 \pm 0,222$ \\
\hline & \multirow{5}{*}{ K-FOLD } & 2 & $0,152 \pm 0,147$ & $0,223 \pm 0,208$ \\
\hline & & 4 & $0,153 \pm 0,148$ & $0,222 \pm 0,211$ \\
\hline & & 5 & $0,151 \pm 0,144$ & $0,223 \pm 0,205$ \\
\hline & & 6 & $0,150 \pm 0,143$ & $0,226 \pm 0,213$ \\
\hline & & 8 & $0,152 \pm 0,145$ & $0,221 \pm 0,206$ \\
\hline
\end{tabular}

\section{B. Conjunto 2: Energia, AAC e LOGD.}

$\mathrm{Na}$ Tab. IV estão relatados os resultados obtidos com a ferramenta de regressão, utilizando como entradas apenas os atributos energia, AAC e LOGD apuradas dos sinais de EMG para cada músculo durante cada um dos 4 exercícios, totalizando $3 \times 4 \times 4=48$ dimensões de entrada para o vetor de atributos do modelo LSSVR. O kernel $\mathrm{RBF}$ apresentou melhor desempenho que os demais na etapa de testes e o kernel polinomial saiu-se melhor na etapa de treino.

TABELA IV. ERROS MÉDIOS OBTIDOS PELO MODELO LSSVR COM SINAL DE EMG PARA CLASSIFICAC̃̃O DO RISCO DE QUEDAS UTILIZANDO OS ATRIBUTOS ENERGIA, AAC E LOGD.

\begin{tabular}{|c|c|c|c|c|}
\hline \multirow{2}{*}{ Kernel } & \multirow{2}{*}{\multicolumn{2}{|c|}{$\begin{array}{c}\text { Cross- } \\
\text { Validation }\end{array}$}} & \multicolumn{2}{|c|}{ ERRO MÉDIO } \\
\hline & & & TREINO & TESTE \\
\hline \multirow{6}{*}{$\begin{array}{c}\mathbf{L} \\
\mathbf{I} \\
\mathbf{N} \\
\mathbf{E} \\
\mathbf{A} \\
\mathbf{R}\end{array}$} & \multicolumn{2}{|c|}{ Leave One Out } & $0,150 \pm 0,143$ & $0,240 \pm 0,229$ \\
\hline & \multirow{5}{*}{ K-FOLD } & 2 & $0,157 \pm 0,154$ & $0,224 \pm 0,215$ \\
\hline & & 4 & $0,150 \pm 0,144$ & $0,225 \pm 0,207$ \\
\hline & & 5 & $0,153 \pm 0,148$ & $0,232 \pm 0,221$ \\
\hline & & 6 & $0,151 \pm 0,143$ & $0,226 \pm 0,214$ \\
\hline & & 8 & $0,151 \pm 0,143$ & $0,225 \pm 0,215$ \\
\hline \multirow{6}{*}{$\begin{array}{c}\mathbf{P} \\
\mathbf{O} \\
\mathbf{L} \\
\mathbf{I} \\
\mathbf{N} \\
\mathbf{O} .\end{array}$} & \multicolumn{2}{|c|}{ Leave One Out } & \multicolumn{2}{|c|}{$\begin{array}{l}\text { Não forneceu resultado numérico } \\
\text { válido }\end{array}$} \\
\hline & \multirow{5}{*}{ K-FOLD } & 2 & $0,143 \pm 0,130$ & $0,226 \pm 0,215$ \\
\hline & & 4 & $0,144 \pm 0,131$ & $0,242 \pm 0,278$ \\
\hline & & 5 & $0,144 \pm 0,131$ & $0,214 \pm 0,198$ \\
\hline & & 6 & $0,143 \pm 0,130$ & $0,215 \pm 0,197$ \\
\hline & & 8 & $0,143 \pm 0,130$ & $0,214 \pm 0,193$ \\
\hline \multirow{6}{*}{$\begin{array}{l}\mathbf{R} \\
\mathbf{B} \\
\mathbf{F}\end{array}$} & \multicolumn{2}{|c|}{ Leave One Out } & $0,145 \pm 0,132$ & $0,197 \pm 0,178$ \\
\hline & \multirow{5}{*}{ K-FOLD } & 2 & $0,145 \pm 0,133$ & $0,196 \pm 0,173$ \\
\hline & & 4 & $0,145 \pm 0,131$ & $0,199 \pm 0,182$ \\
\hline & & 5 & $0,144 \pm 0,131$ & $0,189 \pm 0,162$ \\
\hline & & 6 & $0,145 \pm 0,131$ & $0,199 \pm 0,176$ \\
\hline & & 8 & $0,144 \pm 0,131$ & $0,200 \pm 0,178$ \\
\hline
\end{tabular}

TABELA V. ERROS MÉDIOS OBTIDOS PELO MODELO LSSVR COM SINAL DE FORÇA PARA CLASSIFICAÇÃO DO RISCO DE QUEDAS UTILIZANDO OS ATRIBUTOS ENERGIA, AAC E LOGD.

\begin{tabular}{|c|c|c|c|c|}
\hline \multirow{2}{*}{ Kernel } & \multirow{2}{*}{\multicolumn{2}{|c|}{$\begin{array}{c}\text { Cross- } \\
\text { Validation }\end{array}$}} & \multicolumn{2}{|c|}{ ERRO MÉDIO } \\
\hline & & & TREINO & TESTE \\
\hline \multirow{6}{*}{$\begin{array}{l}\mathbf{L} \\
\mathbf{I} \\
\mathbf{N} \\
\mathbf{E} \\
\mathbf{A} \\
\mathbf{R}\end{array}$} & \multicolumn{2}{|c|}{ Leave One Out } & $0,215 \pm 0,246$ & $0,224 \pm 0,207$ \\
\hline & \multirow{5}{*}{ K-FOLD } & 2 & $0,209 \pm 0,243$ & $0,229 \pm 0,220$ \\
\hline & & 4 & $0,209 \pm 0,244$ & $0,232 \pm 0,223$ \\
\hline & & 5 & $0,209 \pm 0,245$ & $0,230 \pm 0,216$ \\
\hline & & 6 & $0,209 \pm 0,244$ & $0,230 \pm 0,220$ \\
\hline & & 8 & $0,208 \pm 0,241$ & $0,235 \pm 0,226$ \\
\hline \multirow{6}{*}{$\begin{array}{c}\mathbf{P} \\
\mathbf{O} \\
\mathbf{L} \\
\mathbf{I} \\
\mathbf{N} \\
\mathbf{O} .\end{array}$} & \multicolumn{2}{|c|}{ Leave One Out } & $0,151 \pm 0,143$ & $0,659 \pm 0,977$ \\
\hline & \multirow{5}{*}{ K-FOLD } & 2 & $0,157 \pm 0,152$ & $0,999 \pm 1,728$ \\
\hline & & 4 & $0,153 \pm 0,149$ & $0,557 \pm 0,846$ \\
\hline & & 5 & $0,154 \pm 0,149$ & $0,654 \pm 0,993$ \\
\hline & & 6 & $0,152 \pm 0,146$ & $0,512 \pm 0,701$ \\
\hline & & 8 & $0,152 \pm 0,144$ & $0,547 \pm 0,764$ \\
\hline \multirow{6}{*}{$\begin{array}{l}\mathbf{R} \\
\mathbf{B} \\
\mathbf{F}\end{array}$} & \multicolumn{2}{|c|}{ Leave One Out } & $0,160 \pm 0,162$ & $0,246 \pm 0,237$ \\
\hline & \multirow{5}{*}{ K-FOLD } & 2 & $0,172 \pm 0,180$ & $0,240 \pm 0,233$ \\
\hline & & 4 & $0,170 \pm 0,179$ & $0,252 \pm 0,249$ \\
\hline & & 5 & $0,169 \pm 0,177$ & $0,245 \pm 0,239$ \\
\hline & & 6 & $0,164 \pm 0,170$ & $0,249 \pm 0,237$ \\
\hline & & 8 & $0,168 \pm 0,177$ & $0,247 \pm 0,242$ \\
\hline
\end{tabular}

$\mathrm{Na}$ Tab. V estão dispostos os resultados encontrados adotando como entradas os atributos energia, AAC e LOGD calculados a partir dos dados de força para cada um dos 4 exercícios realizados, totalizando $3 \times 4=12$ atributos de entrada para o modelo LSSVR. Novamente, o kernel linear apresentou erros de treino e teste bem próximos, sendo o seu desempenho na testagem melhor quue os demais kernels.

\section{Conjunto 3: AAC e LOGD}

$\mathrm{Na}$ Tab. VI estão relatados os resultados obtidos utilizando como entradas apenas os atributos AAC e LOGD extraídos dos sinais de EMG de cada músculo monitorado durante a realização dos 4 exercícios propostos, totalizando $2 \times 4 \times 4=32$ entradas para o modelo LSSVR. Nesta configuração específica o kernel linear 
apresentou desempenho inferior aos demais kernels nas duas etapas. O kernel RBF apresentou erros de teste menores que o kernel polinomial.

TABELA VI. ERROS MÉDIOS OBTIDOS PELO MODELO LSSVR COM SINAL DE EMG PARA CLASSIFICAÇÃO DO RISCO DE QUEDAS UTILIZANDO OS ATRIBUTOS AAC E LOGD.

\begin{tabular}{|c|c|c|c|c|}
\hline \multirow{2}{*}{ Kernel } & \multirow{2}{*}{\multicolumn{2}{|c|}{$\begin{array}{c}\text { Cross- } \\
\text { Validation }\end{array}$}} & \multicolumn{2}{|c|}{ ERRO MÉDIO } \\
\hline & & & TREINO & TESTE \\
\hline \multirow{6}{*}{$\begin{array}{c}\mathbf{L} \\
\mathbf{I} \\
\mathbf{N} \\
\mathbf{E} \\
\mathbf{A} \\
\mathbf{R}\end{array}$} & \multicolumn{2}{|c|}{ Leave One Out } & $0,146 \pm 0,135$ & $0,207 \pm 0,187$ \\
\hline & \multirow{5}{*}{ K-FOLD } & 2 & $0,149 \pm 0,142$ & $0,210 \pm 0,188$ \\
\hline & & 4 & $0,149 \pm 0,139$ & $0,212 \pm 0,195$ \\
\hline & & 5 & $0,149 \pm 0,142$ & $0,218 \pm 0,198$ \\
\hline & & 6 & $0,147 \pm 0,139$ & $0,208 \pm 0,189$ \\
\hline & & 8 & $0,148 \pm 0,139$ & $0,207 \pm 0,192$ \\
\hline \multirow{6}{*}{$\begin{array}{l}\mathbf{P} \\
\mathbf{O} \\
\mathbf{L} \\
\mathbf{I} \\
\mathbf{N} \\
\mathbf{O} .\end{array}$} & \multicolumn{2}{|c|}{ Leave One Out } & \multicolumn{2}{|c|}{$\begin{array}{c}\text { Não forneceu resultado numérico } \\
\text { válido }\end{array}$} \\
\hline & \multirow{5}{*}{ K-FOLD } & 2 & $0,144 \pm 0,130$ & $0,196 \pm 0,175$ \\
\hline & & 4 & $0,145 \pm 0,131$ & $0,195 \pm 0,173$ \\
\hline & & 5 & $0,144 \pm 0,131$ & $0,200 \pm 0,179$ \\
\hline & & 6 & $0,144 \pm 0,131$ & $0,198 \pm 0,175$ \\
\hline & & 8 & $0,145 \pm 0,131$ & $0,193 \pm 0,175$ \\
\hline \multirow{6}{*}{$\begin{array}{l}\mathbf{R} \\
\mathbf{B} \\
\mathbf{F}\end{array}$} & \multicolumn{2}{|c|}{ Leave One Out } & $0,145 \pm 0,131$ & $0,191 \pm 0,167$ \\
\hline & \multirow{5}{*}{ K-FOLD } & 2 & $0,146 \pm 0,133$ & $0,186 \pm 0,157$ \\
\hline & & 4 & $0,144 \pm 0,131$ & $0,186 \pm 0,159$ \\
\hline & & 5 & $0,145 \pm 0,131$ & $0,191 \pm 0,168$ \\
\hline & & 6 & $0,144 \pm 0,130$ & $0,182 \pm 0,157$ \\
\hline & & 8 & $0,145 \pm 0,132$ & $0,181 \pm 0,154$ \\
\hline
\end{tabular}

Os resultados encontrados utilizando como entradas os atributos AAC e LOGD extraídos dos sinais de força estão dispostos na Tab. VII. Neste caso, o modelo LSSVR contava com um vetor de entrada composto de $2 \times 4=8$ atributos. Novamente, o kernel linear apresentou erros relativamente próximos tanto no treino quanto no teste.

TABELA VII. ERROS MÉDIOS OBTIDOS PELO MODELO LSSVR COM SINAL DE FORÇA PARA CLASSIFICACCÃO DO RISCO DE QUEDAS UTILIZANDO OS ATRIBUTOS AAC E LOGD.

\begin{tabular}{|c|c|c|c|c|}
\hline \multirow{2}{*}{ Kernel } & \multirow{2}{*}{\multicolumn{2}{|c|}{$\begin{array}{c}\text { Cross- } \\
\text { Validation }\end{array}$}} & \multicolumn{2}{|c|}{ ERRO MÉDIO } \\
\hline & & & TREINO & TESTE \\
\hline \multirow{6}{*}{$\begin{array}{l}\mathbf{L} \\
\mathbf{I} \\
\mathbf{N} \\
\mathbf{E} \\
\mathbf{A} \\
\mathbf{R}\end{array}$} & \multicolumn{2}{|c|}{ Leave One Out } & $0,208 \pm 0,240$ & $0,235 \pm 0,230$ \\
\hline & \multirow{5}{*}{ K-FOLD } & 2 & $0,209 \pm 0,242$ & $0,235 \pm 0,228$ \\
\hline & & 4 & $0,209 \pm 0,243$ & $0,231 \pm 0,223$ \\
\hline & & 5 & $0,211 \pm 0,246$ & $0,224 \pm 0,207$ \\
\hline & & 6 & $0,209 \pm 0,243$ & $0,232 \pm 0,221$ \\
\hline & & 8 & $0,210 \pm 0,245$ & $0,228 \pm 0,214$ \\
\hline \multirow{6}{*}{$\begin{array}{c}\mathbf{P} \\
\mathbf{O} \\
\mathbf{L} \\
\mathbf{I} \\
\mathbf{N} \\
\mathbf{O} .\end{array}$} & \multicolumn{2}{|c|}{ Leave One Out } & $0,160 \pm 0,159$ & $0,317 \pm 0,320$ \\
\hline & \multirow{5}{*}{ K-FOLD } & 2 & $0,168 \pm 0,172$ & $0,396 \pm 0,496$ \\
\hline & & 4 & $0,165 \pm 0,168$ & $0,349 \pm 0,397$ \\
\hline & & 5 & $0,164 \pm 0,165$ & $0,341 \pm 0,374$ \\
\hline & & 6 & $0,164 \pm 0,166$ & $0,340 \pm 0,377$ \\
\hline & & 8 & $0,165 \pm 0,168$ & $0,327 \pm 0,341$ \\
\hline \multirow{6}{*}{$\begin{array}{l}\mathbf{R} \\
\mathbf{B} \\
\mathbf{F}\end{array}$} & \multicolumn{2}{|c|}{ Leave One Out } & $0,164 \pm 0,166$ & $0,246 \pm 0,237$ \\
\hline & \multirow{5}{*}{ K-FOLD } & 2 & $0,170 \pm 0,177$ & $0,237 \pm 0,229$ \\
\hline & & 4 & $0,172 \pm 0,180$ & $0,245 \pm 0,240$ \\
\hline & & 5 & $0,172 \pm 0,181$ & $0,242 \pm 0,236$ \\
\hline & & 6 & $0,172 \pm 0,181$ & $0,240 \pm 0,232$ \\
\hline & & 8 & $0,167 \pm 0,174$ & $0,241 \pm 0,232$ \\
\hline
\end{tabular}

\section{DISCUSSÃO}

Uma das adaptações musculoesqueléticas que ocorrem com o processo do envelhecimento é o desenvolvimento de sarcopenia, ou seja, redução da força, massa muscular global e prejuízo no desempenho físico [19]. A fraqueza muscular é frequentemente associada à facilidade de cair $\mathrm{e}$ essa relação tem sido amplamente discutida na literatura $[20,21]$.
Em contrapartida, em todos os experimentos executados neste estudo observou-se uma superioridade do sinal elétrico em relação à força captada pela célula de carga na classificação do risco de quedas, pois as execuções com dados extraídos pela dinamometria apresentaram erros mais elevados, sobretudo na etapa de testes. Isso levanta a hipótese de que o torque talvez não tenha um protagonismo tão sólido na manutenção do equilíbrio quanto se pensava. De fato, um estudo recente prospectivo não identificou associação entre a força muscular do membro inferior dominante e a ocorrência de quedas futuras entre idosos que até então não possuíam histórico de quedas, de forma que é importante identificar outros fatores que possam contribuir para o primeiro episódio de desequilíbrio [22]. Contudo, há também a possibilidade de que os atributos considerados sejam mais apropriados para os sinais de EMG que para os sinais de força. Ressalta-se, ainda, que em todos os testes com dados de força, apesar do modelo LSSVR com kernel $\mathrm{RBF}$ ter tido desempenho pior que o observado nos testes com dados de eletromiografia, os modelos LSSVR com kernel RBF foram capazes de estimar o risco de queda com uma boa acurácia com os dados de força como entrada.

Nos testes realizados não se observou influência do tamanho das partições utilizadas na validação cruzada em relação ao erro gerado. Em relação ao tipo de kernel utilizado, o RBF destacou-se em relação aos demais.

No geral, o modelo LSSVR com kernel RBF, independente do método de treinamento, da quantidade de partições utilizadas na validação cruzada e do tipo de dados de entrada (sinais do EMG ou de força) foi eficaz na classificação do risco de quedas, sendo que as abordagens que usam sinais de EMG saíram-se um pouco melhores. Quando observamos o desempenho considerando a quantidade de entradas fornecidas, observa-se um desempenho muito próximo entre a abordagem que utilizou todos os atributos (máximo, mínimo, média, desvio padrão, RMS, energia, LOGD e AAC) como entradas e aquela que utilizou apenas o LOGD e AAC dos sinais. Assim, considerando o desempenho computacional, seria conveniente utilizar a proposta considerando apenas esses dois atributos.

O kernel polinomial obteve maiores erros de teste na maioria das situações testadas, no entanto Já, quando consideramos dados da eletromiografia, o modelo LSLVR com kernel polinomial obteve um bom desempenho tanto na etapa de treinamento quanto de testes, seguindo o mesmo padrão apresentado pelo modelo LSSVR com kernel RBF, com erros inferiores a 0,4. Já com as entradas obtidas a partir dos sinais de força, a faixa de erro do kernel polinomial foi variável, chegando até próximo de 1. Contudo, considerando que a EBB, usado como padrão ouro nos testes, possui uma pontuação máxima de 56 pontos, um erro médio próximo a 1 ainda é aceitável. Quanto ao uso de kernel linear, observou-se que os modelos LSSVR com este kernel apresentaram desempenho semelhante àqueles com kernel RBF quando as entradas eram obtidas a partir de sinais eletromiográficos.

Para concluir, a principal limitação do presente estudo reside no baixo número de amostras coletadas, devido às restrições sociais impostas pela pandemia de COVID-19 
e, sobretudo, ao fato do público-alvo (idosos) constituir grupo de risco para complicações em caso de infecção pelo SARS-CoV 2.

\section{CONCLUSÕES}

A classificação do risco de quedas em idosos é um importante dado para promover uma melhor assistência à saúde do idoso e por meio dos resultados encontrados neste estudo é possível confirmar a viabilidade da utilização de sinais eletromiográficos e dinamométricos em um algoritmo LSSVR com este fim. Além disso, a análise do sinal elétrico mostrou-se mais efetiva do que a força mensurada por dinamometria, independente do tipo de kernel ou método de validação empregado. No entanto, ressalta-se a importância de se desenvolver novos estudos com uma amostra ampliada. Como trabalhos futuros pretendemos utilizar uma ferramenta de seleção de atributos para selecionar quais são as mais adequadas a serem utilizadas de cada músculo, uma vez que nesta pesquisa consideramos os mesmos atributos simultaneamente para todos os músculos monitorados.

\section{AGRADECIMENTOS}

O presente trabalho foi realizado com apoio da CAPES (Código de Financiamento 001) e do CNPq (No. 309379/2019-9).

\section{REFERÊNCIAS}

11] V.E. Closs and C.H.A Schwanke. "A evolução do índice de envelhecimento no Brasil, nas suas regiões e unidades federativas no período de 1970 a 2010," Rev. Bras. Geriatr. Gerontol., Rio de Janeiro, vol. 15, num. 3, pp. 443-458, 2012

[2] F.V. Siqueira et al. "Prevalence of falls in elderly in Brazil: a countrywide analysis," Cad. Saúde Pública, vol. 27, num. 9, pp. 1819-1826, 2011.

[3] C.S. Florence et al. "The medical costs of fatal falls and fall injuries among older adults," J. Am. Geriatr. Soc., vol.66, num.4, pp.693698, Abril 2018.

[4] J. Zeeh, Y. Reinhardt and H. Heppner. "Stürze im alter," MMWFortschritte der Medizin, vol.159, num.13, pp.52-58, 2017.

[5] J.H. Ansai et al. "Revisão de dois instrumentos clínicos de avaliação para predizer risco de quedas em idosos," Rev. Bras. Geriatr. Gerontol., Rio de Janeiro, vol.17, num.1, pp.177-189, 2014.

[6] B. Blobel, L. Lhotska, P. Pharow and F. Sousa. (Ed.). pHealth 2020. Amsterdã: IOS Press, 2020, pp.176-181. Disponível em: https://ebooks.iospress.nl/volume/phealth-2020-proceedings-of-the17 th-international-conference-on-wearable-micro-and-nanotechnologies-for-personalized-health. Acesso em: 18 março 2021.

[7] S.H. Park. "Tools for assessing fall risk in the elderly: a systematic review and meta-analysis," Aging Clin. Exp. Res., vol.30, num.1, pp.1-16, 2018.

[8] F. Buisseret et al. "Timed up and go and six-minute walking tests with wearable inertial sensor: one step further for the prediction of the risk of fall in elderly nursing home people," Sensors, vol.20, num.11, pp.1-15, 2020.

[9] Lugade V, Lin V, Farley A, Chou, L-S. An Artificial Neural Network Estimation of Gait Balance Control in the Elderly Using Clinical Evaluations. PLoS ONE. vol. 9, num 5, 2014.

[10] Razmara J, Zaboli MH. Elderly fall risk prediction based on a physiological profile approach using artificial neural networks. Health Informatics Journal. pp, 1-9, 2016.

[11] Y. Guo, X. Li, G. Bai, J. Ma. "Time series prediction method based on LS-SVR with modified gaussian RBF," In: International Conference on Neural Information Processing, 19.2012, Berlim. Proceedings, Berlim: Springer, pp.9-17, Novembro 2012.

[12] B.R. Greene et al. Quantitative falls risk estimation through multisensor assessment of standing balance. Physiol Meas. Vol. 33, pp. 2049-2063, 2012.
[13] M.W. Rivolta et al. Evaluation of the Tinetti score and fall risk assessment via accelerometry-based movement analysis. Artificial Intelligence in Medicine. pp. 1-10, 2018.

[14] S. Bounyong. "Fall risk estimation based on co-contraction of lower limb during walking," In: IEEE International Conference on Consumer Electronics,34., 2016, Las Vegas. Proceedings, pp. 331332, 2016.

[15] S.T. Miyamoto, I. Lombardi Junior, K.O. Berg, L.R. Ramos and J. Natour. "Brazilian version of the Berg balance scale," Braz J Med Biol Res., vol.37, num.9, pp.1411-1421, Setembro, 2004.

[16] A. Phinyomark, P. Phukpattaranont and C. Limsakul. "Feature reduction and selection for EMG signal classification," Expert Systems with Applications, vol.39, num.8, pp.7420-7431, 2012.

[17] T. Fukuda. "Root Mean Square Value of the Electromyographic Signal in the Isometric Torque of the Quadriceps, Hamstrings and Brachial Biceps Muscles in Female," Applied Research., vol.10, pp.32-39, 2010.

[18] C.J. De Luca. "The use of surface electromyography in biomechanics," Journal of Applied Biomechanics, vol.13, pp.135163, 1997.

[19] B.P. Martinez, F.W.R. Camelier, A.A. Camelier. "Sarcopenia em idosos: um estudo de revisão," Revista Pesquisa em Fisioterapia, vol.4, num.1, pp.62-70, 2014.

[20] V.S. Borges, N.S. Silva, A.C. Malta, N.C. Xavier and L.E.S. Bernardes. "Falls, muscle strength and functional abilities in community-dwelling elderly women," Fisioter. Mov., vol.30, num.2, pp.357-366, 2017.

[21] A. Ahmadiahangar et al. "The role of quadriceps muscle strength in the development of falls in the elderly people, a cross-sectional study," Chiropractic \& Manual Therapies, pp.26-31, 2018.

[22] J.M. Porto. "Relationship between lower limb muscle strength and future falls among community-dwelling older adults with no history of falls: a prospective 1-year study," Journal of Applied Gerontology, vol.0, num.0, pp.1-8, 2020. 